\section{mTOR inhibition acts as an unexpected checkpoint in p53- mediated tumor suppression}

\author{
Ning Kon, ${ }^{1}$ Yang Ou, ${ }^{1}$ Shang-Jui Wang, ${ }^{1}$ Huan $\mathrm{Li}^{1}{ }^{1}$ \\ Anil K. Rustgi, ${ }^{2}$ and Wei $\mathrm{Gu}^{1,2}$ \\ ${ }^{1}$ Institute for Cancer Genetics, Department of Pathology and Cell
Biology, Columbia University, New York, New York 10032,
USA; ${ }^{2}$ Herbert Irving Comprehensive Cancer Center, College of
Physicians and Surgeons, Columbia University, New York,
New York 10032, USA
}

Here, we showed that the acetylation-defective p53-4KR mice, lacking the ability of cell cycle arrest, senescence, apoptosis, and ferroptosis, were tumor prone but failed to develop early-onset tumors. By identifying a novel p53 acetylation site at lysine K136, we found that simultaneous mutations at all five acetylation sites (p53-5KR) diminished its remaining tumor suppression function. Moreover, the embryonic lethality caused by the deficiency of $m d m 2$ was fully rescued in the background of $p 53^{5 K R / 5 K R}$, but not $p 53^{4 K R / 4 K R}$ background. p53-4KR retained the ability to suppress $m$ TOR function but this activity was abolished in p53-5KR cells. Notably, the early-onset tumor formation observed in $p 53^{5 K R / 5 K R}$ and $p 53$ null mice was suppressed upon the treatment of the mTOR inhibitor. These results suggest that p53-mediated mTOR regulation plays an important role in both embryonic development and tumor suppression, independent of cell cycle arrest, senescence, apoptosis, and ferroptosis.

Supplemental material is available for this article.

Received May 29, 2020; revised version accepted October 21, 2020.

p53 plays a central role by acting as a transcription factor to modulate various types of cellular processes to suppress cancer development (Kastenhuber and Lowe 2017; Kaiser and Attardi 2018). Although the classic activities of p53 including cell cycle arrest, senescence, and apoptosis serve as critical barriers to cancer development, accumulating evidence suggests that loss of p53-dependent cell cycle arrest, apoptosis, and senescence is not sufficient to abrogate the tumor suppression activity of p53 (Brady et al. 2011; Li et al. 2012; Valente et al. 2013). We and others recently found that p53 plays an important role in modulating ferroptotic responses through its metabolic targets (Jiang et al. 2015; Jennis et al. 2016; Ou et al. 2016; Saint-Germain et al. 2017). Ferroptosis, an iron-dependent form of nonapoptotic cell death driven by lipidbased reactive oxygen species (ROS), connects tumor suppression with several different metabolic pathways

[Keywords: p53; acetylation; mTOR; SESN2; DDIT4/REDD1; transcriptional regulation; Mdm2; tumor suppression] Corresponding author: wg8@cumc.columbia.edu

Article published online ahead of print. Article and publication date are online at http://www.genesdev.org/cgi/doi/10.1101/gad.340919.120.
(Stockwell et al. 2017). Indeed, p53-3KR (3KR:K117R+ $\mathrm{K} 161 \mathrm{R}+\mathrm{K} 162 \mathrm{R})$, an acetylation-defective mutant that fails to induce cell cycle arrest, senescence and apoptosis, retains the tumor suppression function and the ability to promote ferroptosis (Jiang et al. 2015). By identifying another acetylation site at lysine K98 in mouse p53 (or K101 for human p53), we found that simultaneous mutations at all four acetylation sites (4KR:K98R + K117R + $\mathrm{K} 161 \mathrm{R}+\mathrm{K} 162 \mathrm{R}$ ) abolished its ferroptosis activity and partially abrogated its tumor suppression function in xenograft tumor models (Wang et al. 2016). Moreover, we identified a novel ALOX12-mediated, GPX4-independent ferroptosis pathway that is critical for the tumor suppression activity of $\mathrm{p} 53$, independent of the classic activities in cell cycle arrest, apoptosis, and senescence (Chu et al. 2019; Stockwell et al. 2020). Nevertheless, it remains unclear whether additional regulatory pathways unrelated to ferroptosis, cell cycle arrest, senescence, and apoptosis contribute to p53-mediated tumor suppression.

\section{Results and Discussion}

p53-4KR mice are tumor--prone but fail to develop early-onset tumors as observed in p53-null mice

To examine whether loss of ferroptosis, cell cycle arrest, apoptosis, and senescence leads to tumor development, a p53-4KR mutant mouse containing four lysine-to-arginine mutations (K98R, K117R, K161R, and K162R) was generated (Supplemental Figs. S1, S2A). The $p 53-4 K R$ heterozygote mice were grossly normal and the intercross of p53-4KR heterozygote mice produced $p 53-4 K R$ homozygote mice at a ratio close to the Mendelian distribution (Supplemental Fig. S2B,C). However, p53-4KR mice developed tumors as they aged, resulting in a median life span of $61 \mathrm{wk}$ for the $p 53-4 K R$ mice (Fig. 1A). Although p53$4 K R$ mice were much more tumor-prone than $p 53-3 K R$ mice, the early-onset tumors were not observed in $p 53$ $4 K R$ mice (Fig. 1A). Most of the tumors developed in p53-4KR mice were lymphomas, including thymic lymphoma (thymoma) (Fig. 1B, panel 5 vs. 1) and B-cell lymphoma (splenomegaly) (Fig. 1B, panel 13 vs. 9). Histological analysis revealed gross disruption of the normal structures of thymus and spleen due to the proliferation of tumor cells (Fig. 1B, panels 2,3 vs. 6,7, and 10,11 vs. $14,15)$. Furthermore, the immunostaining of p53 was positive in the tumor cells, indicating that p53-4KR accumulated during tumorigenesis (Fig. 1B, panel 8 vs. 4 and 16 vs. 12). There were also metastases in the $p 53-4 K R$ mice with tumor burden indicated by the presence of cells in many tissues with intense blue hematoxylin nuclear staining, typically associated with proliferating cells (Fig. 1C, panel 4 vs. 1 and 11 vs. 8). Many of the metastatic cells were positive for p53 staining (Fig. 1C, panel 5 vs. 2 and 12 vs. 9), and ki67 staining (Fig. 1C, panel 6 vs. 3 and 13 vs. 10). Finally, these cells were positive for CD3 staining, indicating that they were T-cells originated from the thymoma

(C) 2021 Kon et al. This article is distributed exclusively by Cold Spring Harbor Laboratory Press for the first six months after the full-issue publication date (see http://genesdev.cshlp.org/site/misc/terms.xhtml). After six months, it is available under a Creative Commons License (Attribution-NonCommercial 4.0 International), as described at http://creativecommons.org/licenses/by-nc/4.0/. 
of this particular mouse (Fig. 1C, panels 7, 14). These data suggest that the loss of p53-mediated ferroptosis, in addition to cell cycle arrest, senescence, and apoptosis promotes tumor development in vivo. Although p53-4KR mice are tumor-prone, they do not develop early-onset tumors as observed in p53-null mice, suggesting that p534KR has yet uncovered tumor suppression activities.

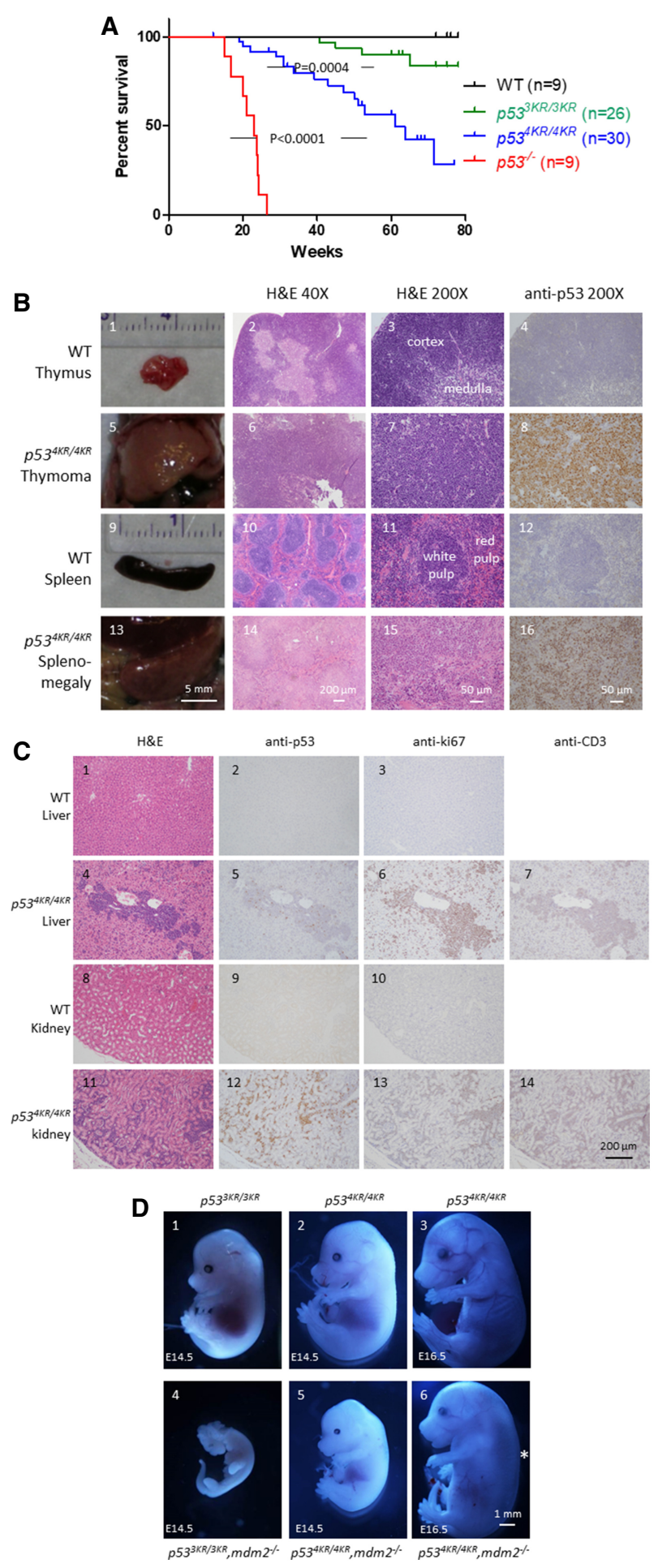

Embryonic lethality caused by mdm2 deficiency is only partially rescued by $p 53-4 K R$ mutant

Our previous study indicated that p53-mediated ferroptosis, like its canonical activities, significantly contribute to embryonic development and the lethality associated with the loss of $m d m 2$ (Jiang et al. 2015). To determine whether the embryonic lethality caused by $m d m 2$ deficiency can be rescued in the background of $p 53^{4 K R / 4 K R}, p 53^{4 K R / 4 K R} /$ $m d m 2^{+/-}$mice were intercrossed to determine whether live $p 53^{4 K R / 4 K R} / \mathrm{mdm} 2^{-/-}$mice can be obtained. No live pups of $p 53^{4 K R / 4 K R} / \mathrm{mdm} 2^{-/-}$were ever recovered from the cross, suggesting that $p 53-4 K R$ mutant cannot fully rescue the embryonic lethality of $m d m 2^{-/-}$mice. To determine whether there was partial rescue of $m d \mathrm{~m}^{-/-}$ mice by the $p 53-4 K R$ mutant, timed breeding was set up to obtain $p 53^{4 K R / 4 K R} / \mathrm{mdm} 2^{-/-}$embryos at various stages of gestation (Supplemental Fig. S3A). Notably, $p 53^{4 K R / 4 K R} / \mathrm{mdm}^{-/-}$embryos displayed a similar developmental characteristics compared with that of the p53 $3^{4 K R / 4 K R}$ embryos at E14.5 (Fig. 1D, panel 5 vs. 2). In comparison, $p 53^{3 K R / 3 K R} / \mathrm{mdm}^{-\gamma}$ - embryos were already dead at E14.5 (Fig. 1D, panel 4 vs. 1). These results suggest that $m d m 2$ knockout embryos were partially rescued by p53-4KR mutant up to E14.5. However, p53 $4 K R / 4 K R /$ $m d m 2^{-/-}$embryos displayed edema at E16.5 (Fig. 1D, panel 6 vs. 3), consistent with cardiovascular defects in these embryos, and died by E18.5. To determine whether p534KR protein can still be regulated by Mdm2, immunostaining of p53 was performed using E14.5 embryonic brains (Supplemental Fig. S3B, panels 1,3). There was modest accumulation of p53-4KR protein in the neuronal cells from the $p 53^{4 K R / 4 K R}$ E14.5 embryo (Supplemental Fig. S3, panel B2). In contrast, the cells in the brain from the E14.5 p53 $3^{4 K R / 4 K R} / \mathrm{mdm}^{-/-}$embryo contained p53 staining ubiquitously, demonstrating that p53-4KR protein was stabilized in the absence of Mdm2 (Supplemental Fig. S3, panel B4). These results suggest that p53-4KR mutant has remnant activities, which cause detrimental defects during embryonic development in the absence of $\mathrm{Mdm} 2$.

\section{K139 acetylation is important for the remaining tumor suppression}

To further elucidate the role of acetylation in modulating p53 function, we searched for additional acetylation sites

Figure 1. $p 53-4 K R$ mice are tumor prone but with a long tumor latency. (A) Kaplan-Meier survival analysis of $p 53-4 K R$ mice with wild-type, $p 53-3 K R$, and $p 53-$ null mice included as controls. Statistical values $(P)$ are indicated. $(B)$ Representative pictures of wild-type thymus (panels 1-4), thymoma from p53-4KR mouse (panels 5-8), wild-type spleen (panels 9-12), and splenomegaly from p53-4KR mouse (panels 13-16). (Panels 1,5,9,13) Gross appearances. (Panels $2,6,10,14)$ Hematoxylin and eosin (H\&E) staining 40×. (Panels $3,7,11,15$ ) H\&E staining 200×. (Panels 4,8,12,16) Immunostaining of p53. (C) Metastases in liver and kidney of a $p 53-4 K R$ mouse with thymoma. (Panels 1-3) Wild-type liver, (Panels 4-7) liver from the p534KR mouse. (Panels 8-10) Wild-type kidney. (Panels 11-14) Kidney from the p53-4KR mouse. (Panels 1,4,8,11) H\&E staining. (Panels $2,5,9,12)$ Immunostaining of p53. (Panels $3,6,10,13$ ) Immunostaining of ki67. (Panels 7,14) Immunostaining of CD3. (D) Pictures of representative embryos. (Panel 1) $p 53^{3 K R / 3 K R}$, E14.5. (Panel 2) $p 53^{4 K R / 4 K R}$, E14.5. (Panel 3) p53 $3^{4 K R / 4 K R}$, E16.5. (Panel 4) $p 53^{3 K R / 3 K R} / \mathrm{mdm}^{-\mathrm{I}^{-}}$


$m d m 2^{-/-}, \mathrm{E} 16.5$. An asterisk indicates edema. 
that may be responsible for the remaining activity of p53. Notably, mass spectrometry analysis of human $\mathrm{p} 53$ protein revealed a new acetylation modification site at K139, which is evolutionarily conserved (Fig. 2A). The K139 lysine residue resides in the DNA-binding domain of p53 and is homologous to the K136 lysine residue in murine p53 (Fig. 2B). To detect acetylation specific at the K139 site, human p53 8KR (K139 wild type, 8KR: K101R + K120R + K164R + $\mathrm{K} 370 \mathrm{R}+\mathrm{K} 372 \mathrm{R}+\mathrm{K} 373 \mathrm{R}+\mathrm{K} 381 \mathrm{R}+\mathrm{K} 382 \mathrm{R}+\mathrm{K} 386 \mathrm{R})$ and 9KR (K139R+8KR) proteins were coexpressed with acetylase Tip60 or CBP, respectively (Fig. 2C). After immunoprecipitation and Western blot assay, no acetylation on p53 was detected in the absence of acetyltransferases (Fig. 2C, lanes 1,2). Coexpression of K139 wild-type p53, but not K139R p53, with Tip60 and CBP resulted in increased levels of K139 acetylation, suggesting that Tip60 and CBP are capable to acetylate p53 at K139 site (Fig. 2C, lanes 3,5 vs. 4,6). Next, we examined the K139 acetylation of endogenous p53 during DNA damage responses. To this end, we treated human osteosarcoma U2OS cells with doxorubicin. Indeed,

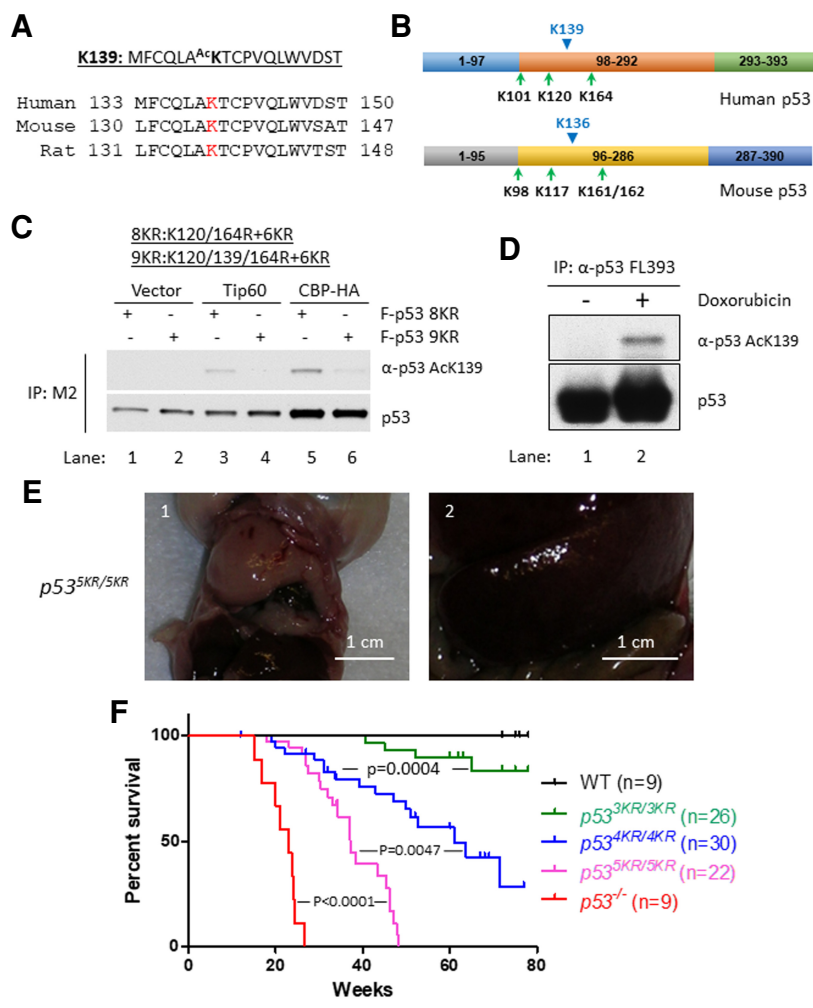

Figure 2. Identification of a novel acetylation site in p53 DNA binding domain and its role in tumor suppression. (A) A new p53 acetylation site K139 was identified by mass spectrometry, which is conserved in human, mouse, and rat $p 53$. (B) Human p53 K139, which is $\mathrm{K} 136$ in mouse p53, locates in DNA-binding domain among the previously identified acetylation sites. $(C)$ Acetylation of p53 K139 by cotransfection with acetyltransferase Tip60 or CBP. After immunoprecipitation, K139 acetylation was detected by Western blot using the anti-p53 acetylated K139 antibody. The total protein levels for p53 was detected by DO-1 antibody. (D) Acetylation on K139 of endogenous $\mathrm{p} 53$ in U2OS cells treated with doxorubicin. After immunoprecipitation, K139 acetylation was detected by Western blot using the anti-p53 acetylated K139 antibody. (E) Representative tumors in p53-5KR mice. (Panel 1) Thymoma. (Panel 2) B-lymphoma. $(F)$ Kaplan-Meier tumor-free survival curves of p53 mutant mice. Statistical values $(P)$ are indicated. the K139 acetylation levels of endogenous p53 were induced upon DNA damage (Fig. 2D, lane 2 vs. 1). Together, these results suggest acetylation on K139 site occurs upon p53 activation in vivo.

To examine the role of K139 acetylation in modulating p53 function in vivo, we generated p53-5KR mutant mouse containing five lysine-to-arginine mutations (K98R, K117R, K136R, K161R, and K162R) (Supplemental Figs. S4, Fig. S5A-C).p53-5KR mice were developmentally normal but tumor prone like $p 53$-null mice. Upon necropsy, most of the $p 53-5 K R$ mice had tumors commonly found in p53-null mice, such as thymoma (Fig. 2E, panel 1) and B-cell lymphomas (Fig. 2E, panel 2). Like p53-4KR, the p53-5KR mutant proteins accumulated during tumorigenesis (Supplemental Fig. S6, panels 2,4,6 vs. 1,3,5). Significantly, the median life-span of the p53-5KR mice decreased to $37 \mathrm{wk}$, compared with $61 \mathrm{wk}$ for the p53-4KR mice, suggesting that p53-5KR mutant has further relinquished the remaining tumor suppression function observed in p53-4KR (Fig. 2F).

Differential effects on mTOR activity by p53 acetylationdeficient mutants

The SESTRIN (SESN1-3) family plays an important role in suppression of mechanistic target of rapamycin kinase complexes 1 and 2 (mTORC1 and mTORC2) (Budanov 2011). It is well established that p53 is able to suppress mTOR activity by activating SESTRIN gene expression (Ellisen et al. 2002; Brugarolas et al. 2004; Feng et al. 2005; Sofer et al. 2005; Budanov and Karin 2008; Olovnikov et al. 2009; Feng and Levine 2010; Budanov 2011; Ding et al. 2019). Interestingly, upon analysis of our RNA-seq data, we found that the mRNA levels of SENS1 and SENS2 were induced upon p53-4KR expression. To ascertain whether SESN1 and SESN2 are indeed regulated by 553 acetylation-defective mutants, the expression of SESN1 and SESN2 was tested using Tet-on-inducible H1299 stable lines expressing mouse p53-3KR, p53-4KR, and p53-5KR (Jiang et al. 2015; Wang et al. 2016). As shown in Figure 3A, induction of the expression of p53-3KR and p53-4KR led to similar increases of mRNA levels of SESN2, whereas the levels of SESN1 induced by p53-4KR were much weaker than the ones induced by p53-3KR. In contrast, the induction of SESN1 and SESN2 was largely abolished using p53-5KR mutant (Fig. $3 \mathrm{~A}$ ). In addition, the levels of DDIT4 (also known as $R E D D 1)$, another known $\mathrm{p} 53$ target involved in repression of mTOR activity (Ellisen et al. 2002; Brugarolas et al. 2004; Sofer et al. 2005), were also induced under the same condition by $\mathrm{p} 53-3 \mathrm{KR}$ and $\mathrm{p} 53-4 \mathrm{KR}$, but not by p53-5KR (Fig. 3A). Next, we tested these findings using $p 53-4 K R$ and $p 53-5 K R$ MEFs. After treating the MEFs with etoposide for $24 \mathrm{~h}$ to induce $\mathrm{p} 53$ activation, the total RNA was extracted, followed by real time-PCR (RT-PCR) assays to determine the relative expression levels of SESN2 and DDIT4. Consistent with the results from the p53-inducible cell lines, the expression of SESN2 and DDIT4 increased after etoposide treatment in p53-4KR MEFs (Fig. 3B). In contrast, the same treatment failed to induce SESN2 and DDIT4 expression in p53-5KR MEFs (Fig. 3B). These results demonstrate that p53-4KR, but not p53-5KR, retains the ability to activate SENS2 and DDIT4.

Since both SENS2 and DDIT4 have been implicated in repressing mTOR activity, we examine whether p53- 


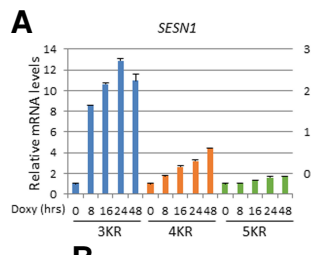

B


C

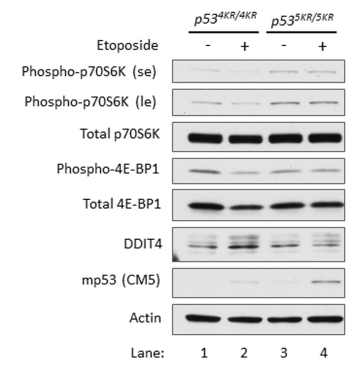

Figure 3. p53-dependent regulation of SESN1, SESN2, and DDIT4, and the effects in suppression of mTOR signaling. (A) The relative mRNA levels of sesn1, sesn2, and DDIT4, determined by RT-PCR assays, in H1299 cells treated with doxycycline for $0,8,16,24$, and $48 \mathrm{~h}$ to express p53 mutants. (B) The relative mRNA levels of sesn 2 and DDIT4 in $p 53^{4 K R / 4 K R}$ and $p 53^{5 K R / 5 K R}$ MEFs treated with etoposide for 0,8 , and $24 \mathrm{~h}$. (C) Effects on mTOR signaling after activation of p53 by treating $p 53-4 K R$ and $p 53-5 K R$ MEFs with etoposide. The whole-cell extracts were analyzed by Western blotting with antibodies as indicated. The levels of actin were used for the protein loading control. (se) Short exposure, (le) long exposure.

4KR and p53-5KR have differential effects in modulating mTOR function. To this end, we monitored the mTOR activity in $p 53-4 K R$ and $p 53-5 K R$ MEFs under normal and DNA-damaging conditions. Both p53 and DDIT4 levels were increased in $p 53-4 K R$ cells upon DNA damage (Fig. $3 \mathrm{C}$, lane 2 vs. 1). Indeed, the levels of phosphorylated p70S6K and 4EBP1, two well-known markers for mTOR activation (Levine et al. 2006; Feng and Levine 2010; Inoki et al. 2012; Saxton and Sabatini 2017; Kim and Guan 2019), were reduced, whereas the total protein levels of both p70S6K and 4EBP1 remained unchanged (Fig. 3C, lane 2 vs. 1). These results implicate that mTOR activity is suppressed upon activation of p53-4KR mutant. In contrast, after the same treatment, p53-5KR failed to induce DDIT4 expression and the levels of phosphorylated p70S6K and 4EBP1 remained unchanged (Fig. 3C, lane 4 vs. 3). These results demonstrate that mTOR signaling is suppressed by p53-4KR, but not by p53-5KR.

p53-5KR is able to fully rescue the embryonic lethality caused by $\mathrm{mdm} 2$ deficiency.

Notably, intercross of $p 53^{5 \mathrm{KR} / 5 \mathrm{KR}} / \mathrm{mdm}^{+/-}$mice generated $p 53^{5 K R / 5 K R} / \mathrm{mdm}^{-/-}$mice, all of which survived well past weaning age (Fig. 4A; Supplemental Fig. S7). Immunostaining of $\mathrm{p} 53$ revealed that $\mathrm{p} 53-5 \mathrm{KR}$ protein was accumulated in many tissues in $p 53^{5 R / 5 K R} / \mathrm{mdm}^{-/-}$mice, such as in spleen, thymus, and testis (Supplemental Fig. S8, panels 1-3), but not in lung and liver (Supplemental Fig. S8, panels 5,6). Taken together, these data indicate that simultaneous mutations at all five acetylation sites (p53-5KR: K136R + 4KR) abrogates the remaining tumor suppression function of p53-4KR and fully rescues the embryonic lethality caused by mdm2 deficiency.
The early-onset tumor formation observed in $\mathrm{p} 53^{5 \mathrm{KR} / 5 \mathrm{KR}}$ and $\mathrm{p} 53-n u l l$ mice is suppressed upon treatment of rapamycin

In contrast to $p 53-5 K R$ mice and $p 53$-null mice, $p 53-4 K R$ mice do not develop early-onset tumors as observed in p53-null mice. We further showed that unlike p53-5KR, p53-4KR retains the ability to suppress mTOR activity. It is very likely that $\mathrm{mTOR}$ signaling is crucial for early-onset tumor formation observed in both $p 53-5 K R$ and p53-null mice. To test this hypothesis, we monitored the tumor development of a cohort of $p 53-5 K R$ mice fed with either control diet or rapamycin diet (42 $\mathrm{mg}$ of rapamycin/ $\mathrm{kg}$ diet) immediately after they were weaned. Interestingly, those on rapamycin diet had lower tumor burden (Fig. 4B, panel 2 vs. 1) and longer survival than the mice on the control diet (Fig. 4C). The median survival for $p 53-5 K R$ mice on rapamycin was significantly longer than the one for $p 53$ $5 K R$ mice on the control diet, $49.5 \mathrm{wk}$ versus $36.8 \mathrm{wk}$ (Fig. 4C). To further demonstrate the significance of inhibiting mTOR activity in p53-mediated tumor suppression, we also treated $p 53$-null mice with a rapamycin diet. Similarly, $p 53$-null mice on the rapamycin diet also had longer survival (36.3 wk) than those on the control diet (23.4 wk; $P$ $=0.003$ ) (Fig. 4D). To explore whether the suppression of
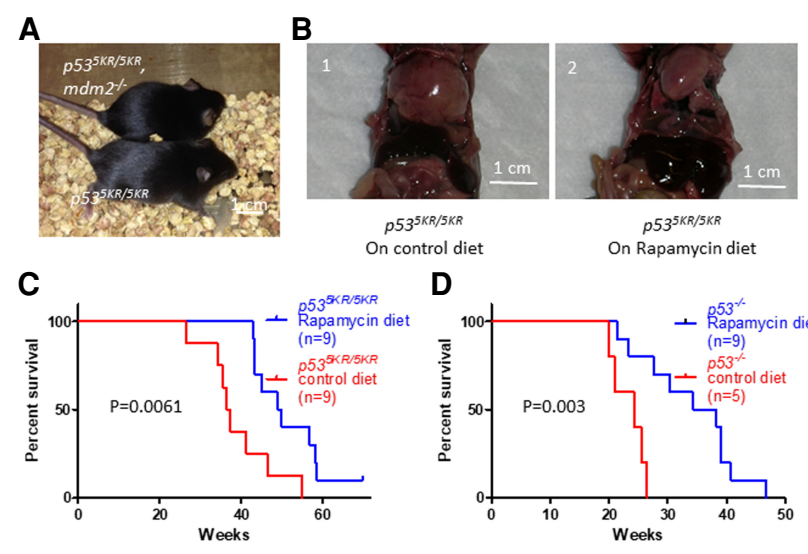

D

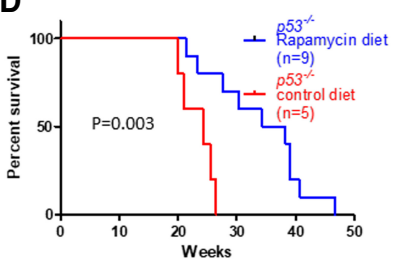

E

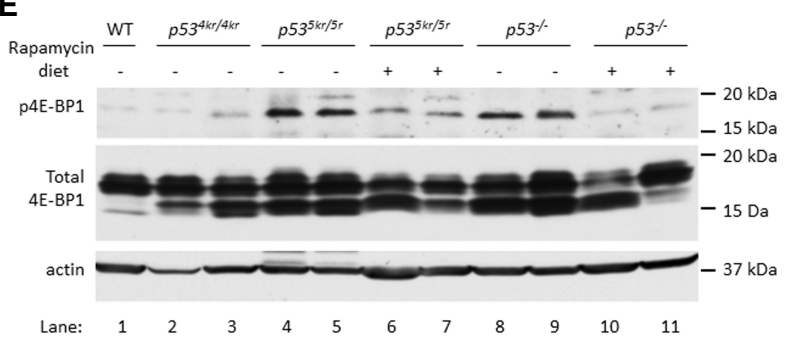

Figure 4. Tumor suppression through inhibiting mTOR signaling. (A) A representative picture of $p 53^{5 K R / 5 K R} / \mathrm{mdm} 2^{--}$and $p 53^{5 K R / 5 K R}$ mice. (B) Representative pictures of thymoma from $p 53^{5 K R / 5 K R}$ mice on control (panel 1) and rapamycin-containing (panel 2) diet. (C) Kaplan-Meier survival curves of $p 53^{5 K R / 5 K R}$ mice on the control and rapamycin containing diet. $(D)$ Kaplan-Meier survival curves of p53-null mice on the control and rapamycin containing diet. Statistical values $(P)$ are indicated. $(E)$ Western blot of the whole-cell extracts from wild-type thymus (lane 1) and from tumor samples of $p 53-4 K R$ mice (lanes 2,3), p53-5KR mice on control diet (lanes 4,5), p53-5KR mice on the rapamycin diet (lanes 6,7), p53-null mice on the control diet (lanes 8,9), and p53-null mice on the rapamycin diet (lanes 10,11) using antibodies of anti-phospho-4E-BP1 and anti-total 4E-BP1. The levels of actin were used as the protein loading control. 
mTOR signaling contributed to the decrease of tumorigenecity in rapamycin-treated mice, markers of mTOR signaling were tested in tumor samples by Western blotting. The results showed that the levels of phosphorylated 4EBP1 was lower in the thymus from wild-type mice and in the tumors from $p 53-4 K R$ mice, compared with those in the tumors from $p 53-5 K R$ and $p 53-$ null mice on the control diet (Fig. 4E, lanes $1-3$ vs. 4,5,8,9). Notably, levels of phospho-4E-BP1 were reduced in tumors from rapamycin-treated $p 53-5 K R$ and $p 53$-null mice (Fig. 4E, lanes 6,7 vs. 4,5 and lanes 10,11 vs. 8,9$)$. These results indicate that mTOR inhibition plays a critical role in modulating the early-onset of tumor formation.

The mTOR protein kinase acts as a central node in coordinating nutrient and growth factor signaling with cell growth. Although numerous studies indicate that p53 is capable of inhibiting mTOR activity through multiple metabolic targets, the precise role of mTOR inhibition in p53-mediated tumor suppression is not well defined. We found that the acetylation-defective mutant p53$4 \mathrm{KR}$, which lacks the ability to undergo p53-mediated cell cycle arrest, senescence, apoptosis, and ferroptosis, retains the ability to inhibit mTOR activity through activating cellular targets such as SENS2 and DITT4, while this activity is completely abolished in p53-5KR. Moreover, the embryonic lethality caused by $m d m 2$ deficiency is only partially rescued in the $p 53^{4 K R / 4 K R}$ background, but fully rescued in the background of $p 53^{5 K R / 5 K R}$. Our results demonstrate that p53-mediated mTOR inhibition is critically involved in both embryonic development and tumor suppression, independent of cell cycle arrest, senescence, apoptosis, and ferroptosis. Notably, although p53-4KR retains its ability in mTOR inhibition, most of $p 53-4 K R$ mice developed tumors with time. Nevertheless, tumorigenesis in $p 53-4 K R$ mice is significantly delayed, compared with p53-5KR mice that lacks the ability to inhibit mTOR. It is very likely that p53-mediated mTOR inhibition plays an important role in modulating early-onset tumor formation but is not sufficient to completely suppress tumor formation. This notion is further validated in p53-null mice upon treatment of rapamycin, a well-known mTOR inhibitor. It is worth noting that despite immense efforts in developing highly potent mTOR inhibitors, most of them lack therapeutic efficacy in vivo (Zaytseva et al. 2012; Fruman and Rommel 2014; Huang et al. 2015). Therefore, our findings suggest that mTOR inhibitors may potentially be used as a novel strategy for delaying tumor development or cancer prevention. Numerous studies have established a central role for mTOR in regulating many fundamental cell processes, from protein synthesis to autophagy. Future studies are clearly needed to investigate whether other unknown activities are still retained by both p53-4KR and p53-5KR mutants and whether p53-mediated mTOR inhibition acts through autophagy, given that autophagy is critically involved in modulating tumor development (Kenzelmann Broz et al. 2013; Simon et al. 2017). Together, our study suggests that p53-mediated mTOR regulation acts as a previously unanticipated checkpoint in both embryonic development and tumor suppression, independent of cell cycle arrest, senescence, apoptosis, and ferroptosis.

\section{Materials and methods}

p53 mutant mice

p53-4KR knockin mouse was generated by gene targeting in mouse embryonic stem cells (Supplemental Fig. S1).The targeting event was confirmed by Southern blot (Supplemental Fig. S2A). After germline transmission and the removal of the neomycin cassette, $p 53-4 K R$ mice were genotyped by PCR assays (Supplemental Fig. S2B,C). Similarly, p53-5KR knockin mice were generated containing K136R and the 4KR mutation (Supplemental Figs. S4, S5A-C). p53-null mice were from the Jackson Laboratory (2101). Maintaining and experimental protocols of mice were approved by the Institutional Animal Care and Use Committee (IACUC) of Columbia University.

\section{Histology and immunostaining}

The tumor samples were processed according to the standard procedures. The antibodies used in immunostaining are anti-p53 (Novocastra CM5), anti-ki67 (Abcam ab15580), and anti-CD3 (Thermo Scientific RM9107). Sagittal sections of the embryos were immunostained using anti-p53 (CM5) antibody and counterstained with hematoxylin.

Tumor-free survival curves

Kaplan-Meier survival curves was composed using GraphPad Prism version 5.00 (GraphPad Software) for Windows.

Protein purification and mass spectrometry

The FLAG-tagged p53 was purified from H1299 cells according to the previous procedure (Li et al. 2012), followed by tryptic digestion and mass spectrometry.

\section{Western blot analysis}

Western blot analysis was done using the whole-cell extracts prepared in RIPA buffer. The blot was probed with antibodies of a polyclonal antibody raised against human p53 K139 acetylated peptide, anti-human p53 (DO-1, Santa Cruz), anti-Phospho-p70 S6 kinase (Thr389; Cell Signaling Technologies), anti-p70 S6 kinase (Cell Signaling Technologies 49D7), anti-phospho-4E-BP1 (Ser65; Cell Signaling Technologies 9451), anti-4E-BP1 (Cell Signaling Technologies 9644), anti-DDIT4 (Proteintech 10638-1-AP), and anti-mouse p53 (Novocastra CM5). The levels of $\beta$-actin (Sigma-Aldrich A3853) were used as the protein loading control.

\section{RNA isolation and real-time (RT)-PCR}

Total RNA was isolated using TRIzol (Invitrogen) and treated with DNase I (Ambion). RT-PCR assay was done according to the manufacturer's protocols. Primers used in the RT-PCR assays were as follows: Sesn 1 (forward $5^{\prime}$-TGTCCCAACGTTTCGTGTC- $3^{\prime}$ and reverse $5^{\prime}$-TGGATAGAGACG ATTCACCAGA-3'), Sesn2 (forward 5'-CAGCGCTTTCATTCCAGTG-3' and reverse 5'-CCGGGTGTAGACCCATCA-3') Ddit4 (forward 5'-CCA GAGAAGAGGGCCTTGA- $3^{\prime}$ and reverse 5'-CCATCCAGGTATGAG GAGTCTT-3').

\section{Manufacturing of rapamycin-containing diet}

Rapamycin was purchased from Rapamycin Holdings. Encapsuled rapamycin (eRapamycin) diet was prepared with eudragit at $42 \mathrm{ppm}$ active rapamycin per kilogram of diet, and the control diet was prepared with the equivalent amount of eudragit by Purina test diet through W.F. Fisher \& Son (Neff et al. 2013; Miller et al. 2014).

\section{Statistical methods}

Results are shown as means \pm S.D. Statistical significance was determined using a two-tailed, unpaired Student; $t$-test or log rank (Mantel-Cox) Test for survival curves.

\section{Acknowledgments}

This work was supported by the National Cancer Institute of the National Institutes of Health under Awards 5R01CA216884, 5R01CA190477, 
5R01CA085533, and 5RO1CA224272 to W.G. This work was also supported by National Institutes of Health grant P01-CA098101 (to A.K.R.). We acknowledge support from the Herbert Irving Comprehensive Cancer Center (HICCC; P30 CA13696) and thank the Molecular Pathology and Proteomics of Shared Resources of HICCC. The content is solely the responsibility of the authors and does not necessarily represent the official views of the National Institutes of Health.

Author contributions: N.K. and W.G. conceived and designed the experiments. N.K., S.-J.W., Y.O., and H.L. performed the methodology and acquired the data. N.K., S.-J.W., Y.O., and H.L. analyzed and interpreted the data. N. K., A.K.R., and W.G. wrote the manuscript.

\section{References}

Brady CA, Jiang D, Mello SS, Johnson TM, Jarvis LA, Kozak MM, Kenzelmann Broz D, Basak S, Park EJ, McLaughlin ME, et al. 2011. Distinct p53 transcriptional programs dictate acute DNA-damage responses and tumor suppression. Cell 145: 571-583. doi:10.1016/j.cell .2011.03.035

Brugarolas J, Lei K, Hurley RL, Manning BD, Reiling JH, Hafen E, Witters LA, Ellisen LW, Kaelin WG Jr. 2004. Regulation of mTOR function in response to hypoxia by REDD1 and the TSC1/TSC2 tumor suppressor complex. Genes Dev 18: 2893-2904. doi:10.1101/gad.1256804

Budanov AV. 2011. Stress-responsive sestrins link p53 with redox regulation and mammalian target of rapamycin signaling. Antioxid Redox Signal 15: 1679-1690. doi:10.1089/ars.2010.3530

Budanov AV, Karin M. 2008. P53 target genes sestrin 1 and sestrin 2 connect genotoxic stress and mTOR signaling. Cell 134: 451-460. doi:10.1016/j .cell.2008.06.028

Chu B, Kon N, Chen D, Li T, Liu T, Jiang L, Song S, Tavana O, Gu W. 2019. ALOX12 is required for p53-mediated tumour suppression through a distinct ferroptosis pathway. Nat Cell Biol 21: 579-591. doi:10.1038/ s41556-019-0305-6

Ding B, Haidurov A, Chawla A, Parmigiani A, van de Kamp G, Dalina A, Yuan F, Lee JH, Chumakov PM, Grossman SR, et al. 2019. p53-inducible SESTRINs might play opposite roles in the regulation of early and late stages of lung carcinogenesis. Oncotarget 10: 6997-7009. doi:10 .18632 /oncotarget. 27367

Ellisen LW, Ramsayer KD, Johannessen CM, Yang A, Beppu H, Minda K, Oliner JD, McKeon F, Haber DA. 2002. REDD1, a developmentally regulated transcriptional target of p63 and p53, links p63 to regulation of reactive oxygen species. Mol Cell 10: 995-1005. doi:10.1016/S10972765(02)00706-2

Feng Z, Levine AJ. 2010. The regulation of energy metabolism and the IGF1/mTOR pathways by the p53 protein. Trends Cell Biol 20: 427-434. doi:10.1016/j.tcb.2010.03.004

Feng Z, Zhang H, Levine AJ, Jin S. 2005. The coordinate regulation of the p53 and mTOR pathways in cells. Proc Natl Acad Sci 102: 8204-8209. doi:10.1073/pnas.0502857102

Fruman DA, Rommel C. 2014. PI3K and cancer: lessons, challenges and opportunities. Nat Rev Drug Discov 13: 140-156. doi:10.1038/nrd4204

Huang Z, Wu Y, Zhou X, Qian J, Zhu W, Shu Y, Liu P. 2015. Clinical efficacy of mTOR inhibitors in solid tumors: a systematic review. Future Oncol 11: 1687-1699. doi:10.2217/fon.15.70

Inoki K, Kim J, Guan KL. 2012. AMPK and mTOR in cellular energy homeostasis and drug targets. Annu Rev Pharmacol Toxicol 52: 381400. doi:10.1146/annurev-pharmtox-010611-134537

Jennis M, Kung CP, Basu S, Budina-Kolomets A, Leu JI, Khaku S, Scott JP, Cai KQ, Campbell MR, Porter DK, et al. 2016. An African-specific polymorphism in the TP53 gene impairs p53 tumor suppressor function in a mouse model. Genes Dev 30: 918-930. doi:10.1101/gad .275891 .115

Jiang L, Kon N, Li T, Wang SJ, Su T, Hibshoosh H, Baer R, Gu W. 2015. Ferroptosis as a 53 -mediated activity during tumour suppression. Nature 520: 57-62. doi:10.1038/nature 14344
Kaiser AM, Attardi LD. 2018. Deconstructing networks of p53-mediated tumor suppression in vivo. Cell Death Differ 25: 93-103. doi:10 $.1038 /$ cdd.2017.171

Kastenhuber ER, Lowe SW. 2017. Putting p53 in context. Cell 170: 1062 1078. doi:10.1016/j.cell.2017.08.028

Kenzelmann Broz D, Spano Mello S, Bieging KT, Jiang D, Dusek RL, Brady CA, Sidow A, Attardi LD. 2013. Global genomic profiling reveals an extensive p53-regulated autophagy program contributing to key p53 responses. Genes Dev 27: 1016-1031. doi:10.1101/gad .212282 .112

Kim J, Guan KL. 2019. mTOR as a central hub of nutrient signalling and cell growth. Nat Cell Biol 21: 63-71. doi:10.1038/s41556-0180205-1

Levine AJ, Feng Z, Mak TW, You H, Jin S. 2006. Coordination and communication between the p53 and IGF-1-AKT-TOR signal transduction pathways. Genes Dev 20: 267-275. doi:10.1101/gad.1363206

Li T, Kon N, Jiang L, Tan M, Ludwig T, Zhao Y, Baer R, Gu W. 2012. Tumor suppression in the absence of p53-mediated cell-cycle arrest, apoptosis, and senescence. Cell 149: 1269-1283. doi:10.1016/j.cell.2012 .04 .026

Miller RA, Harrison DE, Astle CM, Fernandez E, Flurkey K, Han M, Javors MA, Li X, Nadon NL, Nelson JF, et al. 2014. Rapamycin-mediated lifespan increase in mice is dose and sex dependent and metabolically distinct from dietary restriction. Aging Cell 13: 468-477. doi:10.1111/acel .12194

Neff F, Flores-Dominguez D, Ryan DP, Horsch M, Schröder S, Adler T, Afonso LC, Aguilar-Pimentel JA, Becker L, Garrett L, et al. 2013. Rapamycin extends murine lifespan but has limited effects on aging. J Clin Invest 123: 3272-3291. doi:10.1172/JCI67674

Olovnikov IA, Kravchenko JE, Chumakov PM. 2009. Homeostatic functions of the p53 tumor suppressor: regulation of energy metabolism and antioxidant defense. Semin Cancer Biol 19: 32-41. doi:10.1016/j semcancer.2008.11.005

Ou Y, Wang SI, Li D, Chu B, Gu W. 2016. Activation of SAT1 engages polyamine metabolism with p53-mediated ferroptotic responses. Proc Nat1 Acad Sci 113: E6806-E6812. doi:10.1073/pnas.1607152113

Saint-Germain E, Mignacca L, Vernier M, Bobbala D, Ilangumaran S, Ferbeyre G. 2017. SOCS1 regulates senescence and ferroptosis by modulating the expression of p53 target genes. Aging 9: 2137-2162. doi:10 .18632/aging.101306

Saxton RA, Sabatini DM. 2017. mTOR signaling in growth, metabolism, and disease. Cell 168: 960-976. doi:10.1016/j.cell.2017.02.004

Simon HU, Friis R, Tait SW, Ryan KM. 2017. Retrograde signaling from autophagy modulates stress responses. Sci Signal 10: eaag2791. doi:10.1126/scisignal.aag2791

Sofer A, Lei K, Johannessen CM, Ellisen LW. 2005. Regulation of mTOR and cell growth in response to energy stress by REDD1. Mol Cell Biol 25: 5834-5845. doi:10.1128/MCB.25.14.5834-5845.2005

Stockwell BR, Friedmann Angeli JP, Bayir H, Bush AI, Conrad M, Dixon SJ, Fulda S, Gascón S, Hatzios SK, Kagan VE, et al. 2017. Ferroptosis: a regulated cell death nexus linking metabolism, redox biology, and disease. Cell 171: 273-285. doi:10.1016/j.cell.2017.09.021

Stockwell BR, Jiang X, Gu W. 2020. Emerging mechanisms and disease relevance of ferroptosis. Trends Cell Biol 30: 478-490. doi:10.1016/j.tcb .2020.02.009

Valente LJ, Gray DH, Michalak EM, Pinon-Hofbauer J, Egle A, Scott CL, Janic A, Strasser A. 2013. p53 efficiently suppresses tumor development in the complete absence of its cell-cycle inhibitory and proapoptotic effectors p21, Puma, and Noxa. Cell Rep 3: 1339-1345. doi:10 .1016/j.celrep.2013.04.012

Wang SJ, Li D, Ou Y, Jiang L, Chen Y, Zhao Y, Gu W. 2016. Acetylation Is crucial for p53-mediated ferroptosis and tumor suppression. Cell Rep 17: 366-373. doi:10.1016/j.celrep.2016.09.022

Zaytseva YY, Valentino JD, Gulhati P, Evers BM. 2012. mTOR inhibitors in cancer therapy. Cancer Lett 319: 1-7. doi:10.1016/j.canlet .2012 .01 .005 


\section{CORRIGENDUM}

Genes \& Development 35: 59-64 (2021)

\section{Corrigendum: mTOR inhibition acts as an unexpected checkpoint in p53-mediated tumor suppression}

Ning Kon, Yang Ou, Shang-Jui Wang, Huan Li, Anil K. Rustgi, and Wei Gu

In the print edition of the above-mentioned article, there were errors in panels 1, 5, 9, and 13 in Figure 1B. These errors have been corrected in the article online.

doi: $10.1101 / \operatorname{gad} .348236 .121$ 


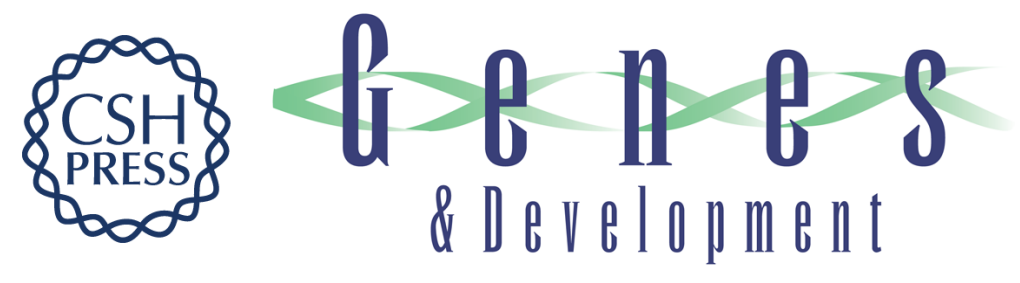

\title{
mTOR inhibition acts as an unexpected checkpoint in p53-mediated tumor suppression
}

\author{
Ning Kon, Yang Ou, Shang-Jui Wang, et al.
}

Genes Dev. 2021, 35: originally published online December 10, 2020

Access the most recent version at doi:10.1101/gad.340919.120

\section{Supplemental http://genesdev.cshlp.org/content/suppl/2020/12/10/gad.340919.120.DC1 \\ Material}

Related Content

Corrigendum: mTOR inhibition acts as an unexpected checkpoint in p53-mediated tumor suppression

Ning Kon, Yang Ou, Shang-Jui Wang, et al.

Genes Dev. February , 2021 35: 300

References This article cites 33 articles, 8 of which can be accessed free at: http://genesdev.cshlp.org/content/35/1-2/59.full.html\#ref-list-1

Articles cited in: http://genesdev.cshlp.org/content/35/1-2/59.full.html\#related-urls

Creative This article is distributed exclusively by Cold Spring Harbor Laboratory Press for the first Commons six months after the full-issue publication date (see

License http://genesdev.cshlp.org/site/misc/terms.xhtml). After six months, it is available under a Creative Commons License (Attribution-NonCommercial 4.0 International), as described at http://creativecommons.org/licenses/by-nc/4.0/.

Email Alerting Receive free email alerts when new articles cite this article - sign up in the box at the top Service right corner of the article or click here.

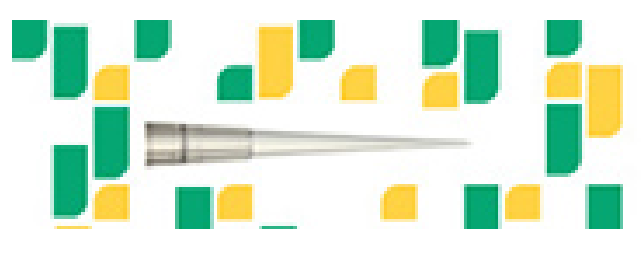

Focused on your science. 